\title{
Dynamical Origin of Low-Frequency Variability in a Highly Nonlinear Midlatitude Coupled Model
}

\author{
S. KRAVTSOV \\ Department of Mathematical Sciences, University of Wisconsin-Milwaukee, Milwaukee, Wisconsin, and \\ Department of Atmospheric and Oceanic Sciences, and Institute of Geophysics and Planetary Physics, University of California, \\ Los Angeles, Los Angeles, California \\ P. BERLOFF \\ Physical Oceanography Department, Woods Hole Oceanographic Institution, Woods Hole, Massachusetts \\ W. K. Dewar \\ Department of Oceanography, The Florida State University, Tallahassee, Florida
}

\section{GHIL}

Départment Terre-Atmosphère-Océan, and Laboratoire de Météorologie Dynamique (CNRS and IPSL), Ecole Normale Supérieure, Paris, France, and Department of Atmospheric and Oceanic Sciences, and Institute of Geophysics and Planetary Physics, University of California, Los Angeles, Los Angeles, California

\section{J. C. McWilliams}

Department of Atmospheric and Oceanic Sciences, and Institute of Geophysics and Planetary Physics, University of California, Los Angeles, Los Angeles, California

(Manuscript received 18 August 2005, in final form 21 April 2006)

\begin{abstract}
A novel mechanism of decadal midlatitude coupled variability, which crucially depends on the nonlinear dynamics of both the atmosphere and the ocean, is presented. The coupled model studied involves quasigeostrophic atmospheric and oceanic components, which communicate with each other via a constant-depth oceanic mixed layer. A series of coupled and uncoupled experiments show that the decadal coupled mode is active across parameter ranges that allow the bimodality of the atmospheric zonal flow to coexist with oceanic turbulence. The latter is most intense in the regions of inertial recirculation (IR). Bimodality is associated with the existence of two distinct anomalously persistent zonal-flow modes, which are characterized by different latitudes of the atmospheric jet stream. The IR reorganizations caused by transitions of the atmosphere from its high- to low-latitude state and vice versa create sea surface temperature anomalies that tend to induce transition to the opposite atmospheric state. The decadal-interdecadal time scale of the resulting oscillation is set by the IR adjustment; the latter depends most sensitively on the oceanic bottom drag. The period $T$ of the nonlinear oscillation is $7-25 \mathrm{yr}$ for the range of parameters explored, with the most realistic parameter values yielding $T \approx 20 \mathrm{yr}$.

Aside from this nonlinear oscillation, an interannual Rossby wave mode is present in all coupled experiments. This coupled mode depends neither on atmospheric bimodality, nor on ocean eddy dynamics; it is analogous to the mode found previously in a channel configuration. Its time scale in the model with a closed ocean basin is set by cross-basin wave propagation and equals $3-5 \mathrm{yr}$ for a basin width comparable with the North Atlantic.
\end{abstract}

Corresponding author address: Dr. Sergey Kravtsov, Department of Mathematical Sciences, University of Wisconsin-Milwaukee, P.O. Box 413, Milwaukee, WI 53201.

E-mail: kravtsov@uwm.edu 


\section{Introduction}

\section{a. Motivation}

A major ambiguity in prescribing any portion of the climate change to midlatitude coupled dynamics stems from apparent failure of the general circulation models (GCMs) to detect a robust and statistically significant atmospheric response to weak, ocean-induced sea surface temperature (SST) anomalies (Kushnir and Held 1996; Saravanan 1998; Rodwell et al. 1999; Mehta et al. 2000). We hypothesize here that, in order to be conducive to midlatitude coupled variability, an atmospheric model must necessarily be characterized by a strongly nonlinear behavior, which will allow small perturbations of external (SST) forcing to cause substantial lowfrequency reorganizations of atmospheric flow patterns. A conceptual way to think about GCMs not supporting midlatitude coupled modes would then be to say that they likely operate in a linear regime (which may be quite realistic, of course). Another feature of GCMs that reduces their potential for active midlatitude coupled dynamics, and can also, in this case, be more easily criticized from a dynamical perspective, has to do with coarse resolutions of the GCMs' oceanic components, a consequence of which is the appearance of relatively smooth, laminar ocean circulations. The ocean, though, is characterized by energetic variability and nonlinear behavior. The clear significance of such turbulence in the ocean calls into question the results of coupled GCM results conducted in the absence of these dynamics.

To address the above hypotheses, we study the behavior of a coupled ocean-atmosphere numerical model, in which both atmospheric and oceanic components are governed by quasigeostrophic (QG) dynamics at spatial resolutions that allow vigorous intrinsic variability in both fluids. In the ocean, this variability is concentrated and near the eastward jet formed by the merger of the separated western boundary currents and the adjacent inertial recirculation (IR) region (see Holland 1978; we use essentially the same model for our experimentation). This region is characterized by two strong vortices of opposite sign, on either side of the separated eastward jet, whose transports exceed those in the Sverdrup interior by a factor of 3-4. A feature of the atmospheric component of the model (which is identical to that of Kravtsov et al. 2005a) of considerable importance to the present study is that in certain parameter ranges of the surface drag coefficient $k$, the model's intrinsic low-frequency variability (LFV) consists of irregular transitions between two anomalously persistent, high- and low-latitude jet states, which we will refer to as the atmospheric bimodality.

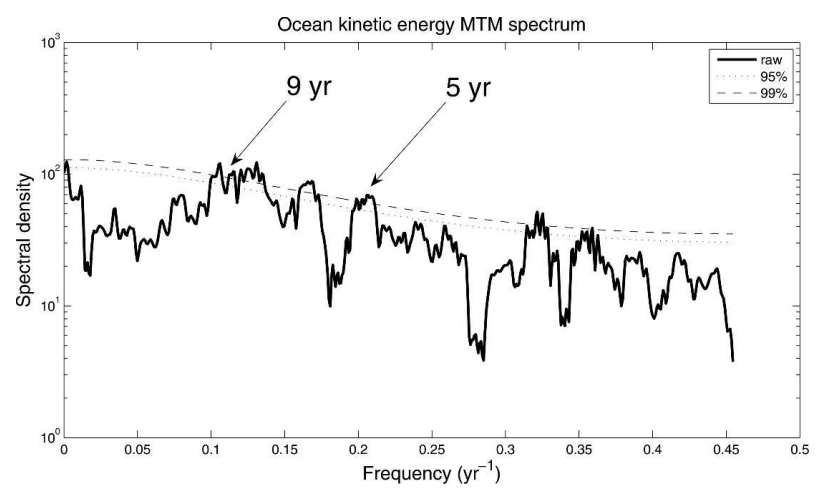

FIG. 1. MTM spectra of ocean kinetic energy: control run (experiment 1).

In our control case, we will use the value of $k^{-1}=$ 6.17 days, for which the intrinsic transitions to the atmospheric low-latitude state are frequent, but irregular in the uncoupled atmospheric setting (see Kravtsov et al. 2005a). In our coupled model, however, where the ocean and atmosphere exchange heat an momentum via a constant-depth mixed layer of Kravtsov and Robertson (2002), the frequency of these transitions varies in time and exhibits a broad spectral peak centered at about 9 yr (not shown); the same spectral peak is present in the oceanic time series (see Fig. 1). The oscillation involves the development of an intense ocean eastward jet and vigorous IR vortices when the highlatitude atmospheric jet persists, while the oceanic jet weakens and breaks down into eddies during the phase characterized by more frequent atmospheric transitions to the low-latitude state (Kravtsov et al. 2006, hereafter K06).

The purpose of the present paper is to investigate the dynamics of this behavior by carrying out and analyzing various uncoupled integrations and studying the sensitivity to oceanic and atmospheric parameters, most notably to the atmospheric surface drag coefficient and the oceanic bottom drag, as well as to the ocean model's horizontal resolution. The former, as we have already pointed out, controls atmospheric bimodality, while the latter two impose restrictions on the oceanic turbulence. The suite of the proposed experiments is thus designed to establish (i) whether the behavior is inherently coupled, and (ii) whether the atmospheric bimodality and oceanic turbulence are essential for this behavior.

\section{b. Summary of experiments and road map}

We summarize the major experiments we have performed in Table 1. Experiment 1 is the control run, 


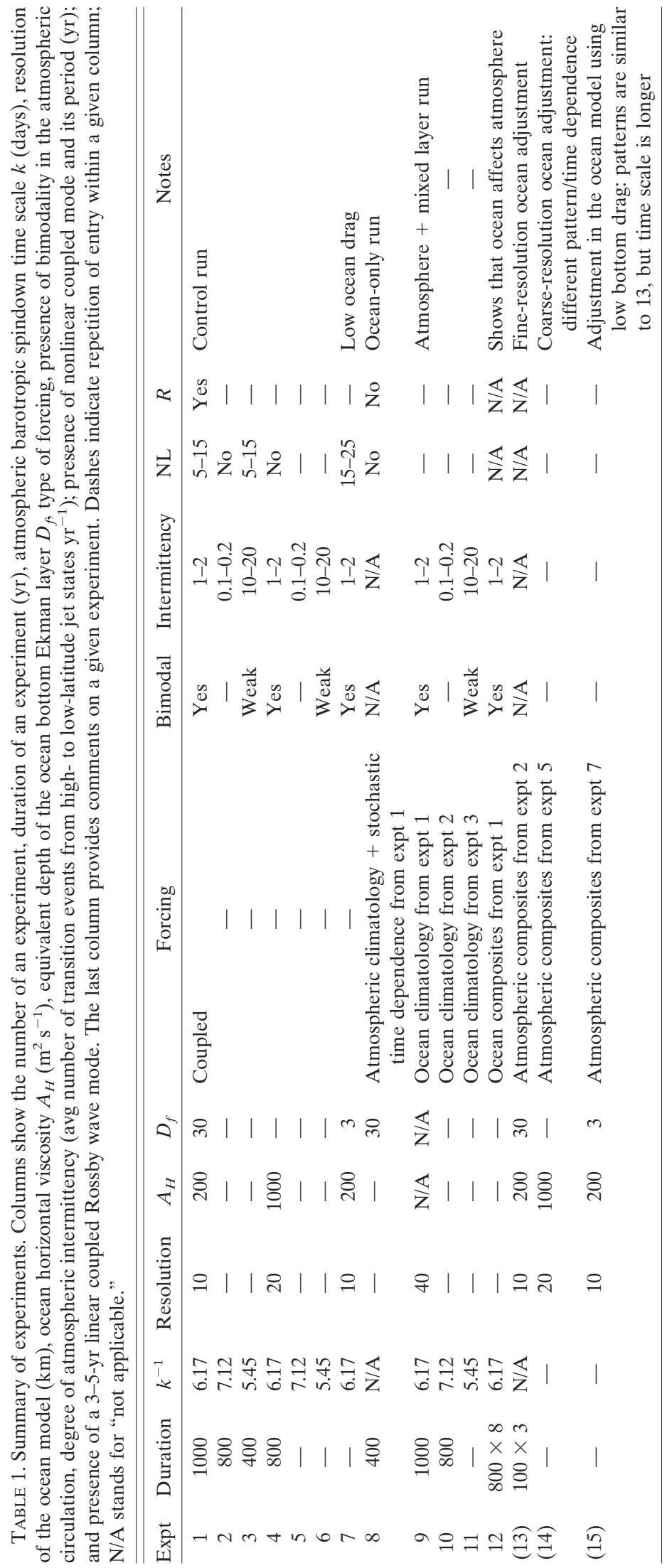


which uses a high value of oceanic bottom drag (an equivalent depth of bottom Ekman layer $D_{f}=30 \mathrm{~m}$ ) and an intermediate atmospheric surface drag of $k^{-1}=$ 6.17 days. Experiments 2 and 3 are identical to experiment 1 except for the value of the atmospheric surface drag, which is respectively lower and higher than that in the control run. Experiments 4-6 are analogous to experiments $1-3$, but use a coarser-resolution ocean model and, hence, higher horizontal viscosity, while experiment 7 uses the resolution and viscosity of the control run, but a value of the ocean bottom drag coefficient that is 10 times smaller $\left(D_{f}=3 \mathrm{~m}\right)$. Thus, experiments 1-7 all use a fully coupled model. We will see, however, that coarse-resolution experiments do not support the coupled mode of the control run, while the period of this mode increases as the ocean bottom drag is reduced. Both of these properties suggest that the ocean eddies are essential to the oscillation.

The remainder of the experiments are uncoupled. Experiments 8-11 are designed to establish the coupled nature of the phenomenon under consideration. Experiment 8 is an ocean-only integration forced by surrogate atmospheric pumping, which consists of a constant, time-mean field and a stochastic component obtained by fitting a linear stochastic model (Kravtsov et al. 2005b) to the history of this forcing from the fully coupled run (experiment 1). Experiments 9-11 use time-mean ocean circulation from experiments $1-3$, respectively, to force the atmosphere-ocean-mixed layer model. The decadal oscillatory behavior is not realized in any of the above uncoupled integrations, and we conclude that coupled dynamics are at play [a spectral peak at a period of about 4-5 yr appears, though, in all coupled experiments (see, e.g., Fig. 1), and is associated with a coupled Rossby wave mode (see section 5)].

To show how the atmosphere responds to oceaninduced SST anomalies, Experiment 12 uses composites of ocean circulation keyed to phase categories of the 9 -yr cycle of experiment 1 (see section $2 b$ ) to force the atmosphere-ocean-mixed layer model and documents changes in conditional probability of the atmospheric low-latitude state. Finally, to clarify the role of ocean eddies in setting up the coupled oscillation's time scale, ocean adjustment experiments 13-15 use oceanQG-interior coupled to an ocean-mixed layer model forced by composites of atmospheric circulation in the high-latitude state for $100 \mathrm{yr}$, the low-latitude state for another $100 \mathrm{yr}$, and, again, $100 \mathrm{yr}$ of the high-latitude state. (These experiments used models from experiment 2, 5, and 7, respectively. Atmospheric composites were computed as described in section 2b.) They will demonstrate that (i) the major difference between the adjustment of the high-resolution, eddy-rich ocean (ex- periment 13) versus its coarse-resolution counterpart (experiment 14) is the lack of eddy-induced SST anomalies in the latter; and (ii) that the oscillation period (in experiments 1 and 7) scales as the eddy-driven adjustment time scale (as determined by experiment 13 and 15 , respectively).

The paper is organized as follows. In section 2, we describe the analysis methods we used. Sections 3 and 4 focus on the roles of atmospheric and oceanic nonlinearity in the coupled oscillation, respectively. The linear coupled Rossby wave mode is described in section 5. Concluding remarks follow in section 6 .

\section{Methods}

\section{a. Spectral analysis}

We applied to the oceanic and atmospheric time series two complementary methods of advanced spectral analysis (Ghil et al. 2002a): the multitaper method (MTM; Thomson 1982, 1990; Mann and Lees 1996) and singular spectrum analysis (SSA; Vautard and Ghil 1989; Dettinger et al. 1995). These methods provide more accurate and reliable detection of periodicity in a given time series compared to traditional Fourier methods, and SSA also provides more consistent compositing procedures.

\section{1) MTM}

MTM replaces the single window used in Fourier analysis by a small set of optimal windows (tapers) that objectively minimize power leakage and reduce uncertainties in the estimated spectra. Statistical significance against a red noise null hypothesis is assessed by fitting an autoregressive $[\mathrm{AR}(1)]$ process to the time series being tested (Mann and Lees 1996). To concentrate on decadal variability, we first took 1-yr-long nonoverlapping box-car averages of a given time series and used three tapers to compute MTM spectra, resulting in a spectral resolution of 0.02 cycle $\mathrm{yr}^{-1}$.

\section{2) SSA}

SSA computes the eigenvalues and eigenvectors of a given time series lag-covariance matrix. These eigenvalues and eigenvectors are also called the singular values of the time series and the temporal empirical orthogonal functions (T-EOFs), respectively. The projection of the original time series onto T-EOFs yields temporal principal components (T-PCs). An oscillatory component is represented in SSA by a single pair of approximately equal singular values, with respective temporal EOFs and PCs in phase quadrature (Vautard 
and Ghil 1989); its characteristic frequency is estimated by maximizing the correlation with a sinusoid. To eliminate spurious pairs, we have applied three tests: (i) a $\chi^{2}$ test against a red noise null hypothesis (Allen and Smith 1996); (ii) a lag-correlation test to verify that two given PCs are indeed in quadrature (Ghil and Mo 1991); and (iii) the "same frequency" and "strong FFT" tests of Vautard et al. (1992). In each case, we have used the 40-day binning and applied an SSA window width of $365 \times 40$ days $=40 \mathrm{yr}$.

The same statistically significant oscillations were detected in most cases when we applied a generalization of SSA to a time series of vectors, the so-called multichannel SSA (M-SSA; Keppenne and Ghil 1993; Plaut and Vautard 1994; Ghil et al. 2002a), to the combined atmospheric and ocean time series. We also computed the reconstructed components (RCs) of the oscillation. The RCs are narrowband versions of the time series, where the band filters are derived from the time series itself in order to maximize the variance captured (Ghil and Vautard 1991; Vautard et al. 1992).

For each time series we have considered, the two complementary methods of spectral analysis described above have identified the same statistically significant periodicities.

\section{b. Compositing}

For each detected oscillation, we obtained its composite cycle in a given oceanic or atmospheric scalar quantity or field by dividing the RC time series obtained by M-SSA into eight phases, and averaging each field under consideration over the days belonging to each phase. The time series we used for defining the eight phases were, typically, the atmospheric jet position and the oceanic kinetic energy, and each of the eight phases contained the same amount of data points.

We applied the same compositing methodology to obtain atmospheric high- and low-latitude jet regime patterns for the use in ocean-only adjustment experiments. In this case, we first computed the probability density function (PDF) of the atmospheric jet's latitude by binning its values into 30 equal segments and counting the number of days the model spent within each segment, divided by the total number of data points in the time series. The PDF was typically strongly skewed and well represented as a sum of two Gaussians. The atmospheric regimes were computed by compositing the points in the neighborhood of each of the two Gaussians.

\section{c. Lagged covariance analysis}

We will use this analysis in section 5 to describe the model's coupled Rossby wave mode. In this approach, we regress the model fields onto the time series of ocean kinetic energy, centered, normalized, and filtered in the 1-10-yr band; multiplying the filtered time series so obtained by -1 has the field at lag 0 correspond to an ocean state with minimum kinetic energy. The convention we use is that the fields at negative lags are the patterns that arise prior to the minimum of the kinetic energy and those at positive lags follow the minimum of kinetic energy.

\section{Nonlinear atmospheric sensitivity}

In this section, we explore dependence of the coupled decadal-interdecadal mode on atmospheric bimodality and establish the ways in which the ocean affects atmospheric circulation nonlinearly, by changing the attractor basin of the atmosphere's low-latitude state.

\section{a. Sensitivity to atmospheric surface friction}

The PDFs of atmospheric jet position for the coupled experiments using lower and higher values of surface friction (experiments 2 and 3 in Table 1) are shown in Fig. 2. The PDF based on data from experiment 2 exhibits pronounced bimodality, while there is considerable skewness, but no multiple PDF maxima in experiment 3; the control run's (experiment 1) PDF has the skewness in between the values characterizing experiments 2 and 3. This skewness is still indicative of the presence of two dynamically distinct states, or modes, namely high- and low-latitude atmospheric states (see Kravtsov et al. 2005a).

An MTM-based spectral analysis of ocean kinetic energy time series from experiments 2 and 3 is shown in Fig. 3. This analysis does identify both the 4- and 10-yr peaks for experiment 3 and a similar 4-yr peak, but no decadal peak in experiment 2 . The decadal peak in experiment 3 is slightly shifted with respect to the 9-yr peak in the control run (experiment 1). Similarly, interannual peak in the control run at $5 \mathrm{yr}$ is slightly shifted with respect to 4 -yr peaks of experiments 2 and 3 .

The interannual peak with a period of $4-5 \mathrm{yr}$ is, in fact, present in all coupled experiments listed in Table 1 , as identified by both MTM and SSA analysis of the oceanic kinetic energy (not shown). In contrast, no uncoupled integration exhibits such a peak (see Table 1). This signal is associated with a coupled propagating Rossby wave, which will be described in greater detail in section 5. This wave does not induce significant variations of the atmospheric jet position, hence none of the spectra of the latter quantity exhibit such a peak (not shown). An interannual spectral peak in the atmo- 


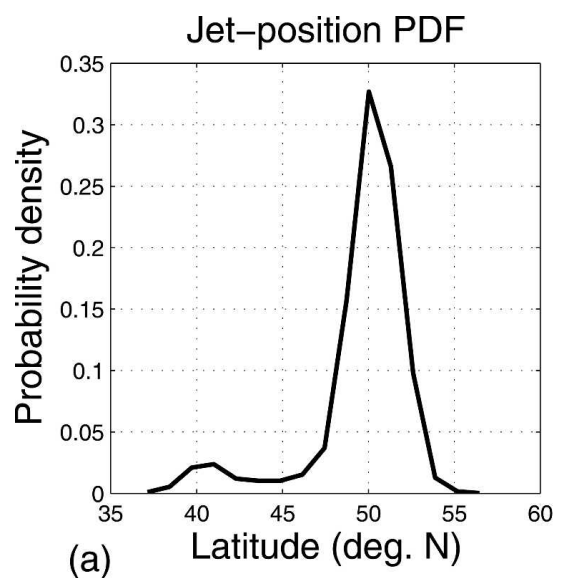

FIG. 2. PDF of atmospheric jet position: (a) experiment 2 in Table 1 (slow spindown); and (b) experiment 3 in Table 1 (fast spindown).

(b) Latitude (deg. N) spheric circulation is, however, captured by applying first traditional PC analysis in space (Preisendorfer 1988) to the monthly mean atmospheric streamfunction. The resulting EOFs 2 and 3 have a wave- 4 spatial pattern and the associated PCs exhibit a $4-5$-yr spectral peak (not shown).
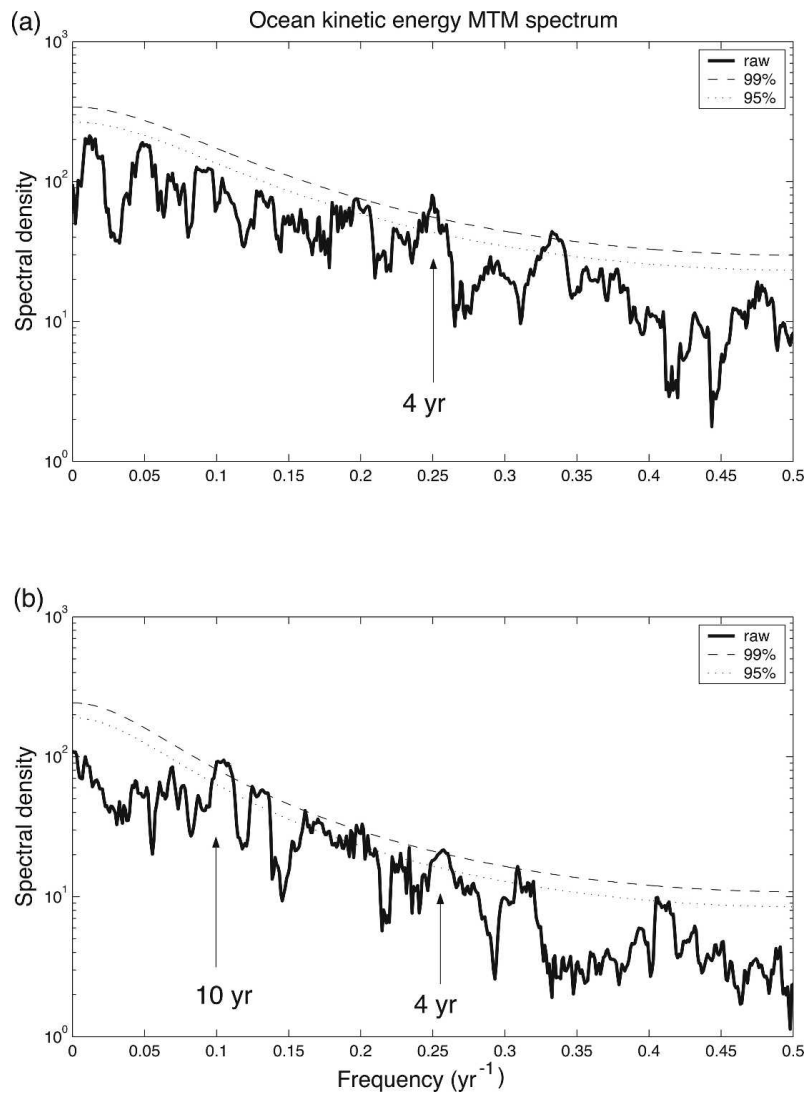

FIG. 3. MTM spectra of ocean kinetic energy: (a) experiment 2 (slow spindown); and (b) experiment 3 (fast spindown).

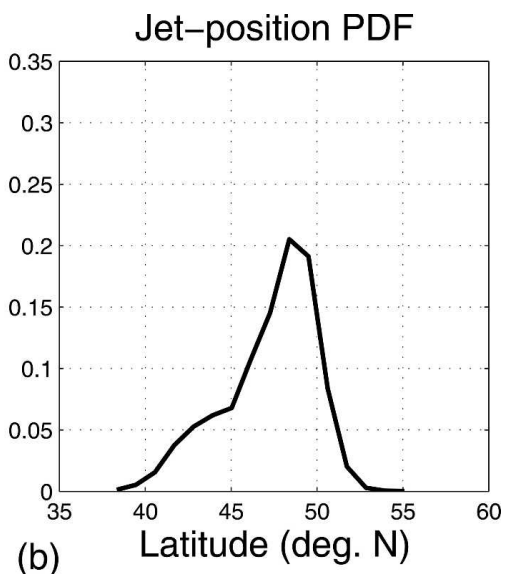

An analysis of experiment 3 (high surface drag), similar to that performed in K06 for the control run, shows that the decadal oscillation identified in the spectra of Figs. 1 and $3 \mathrm{~b}$ here has the same spatial and temporal characteristics (not shown) as the decadal oscillation of the control run, although it is responsible for a smaller fraction of the model's variance. As the surface friction parameter increases further and the atmospheric model becomes less and less bimodal (see Kravtsov et al. 2005a), the coupled nonlinear oscillation disappears altogether, along with nonlinear atmospheric sensitivity to ocean-induced SST anomalies.

The absence of the coupled nonlinear oscillation from experiment 2 with low atmospheric surface drag (Fig. 3a) is somewhat surprising in light of our arguments about the role of atmospheric bimodality in this oscillation. The apparent contradiction has to do with the number of atmospheric jet transitions to the lowlatitude state at low values of bottom drag. Figures $4 a-c$ show 200-yr-long segments of the atmospheric jet position simulated in experiments 2,1 , and 3 , respectively.

For low values of surface friction (Fig. 4a), the time series is extremely intermittent, with very infrequent transitions to the low-latitude state, at a rate of roughly one transition per decade. This characteristic of the experiments is summarized in the "intermittency" column of Table 1. As a consequence of these transitions being so rare, there is little opportunity for the ocean to influence their frequency on decadal time scales, and the coupled decadal oscillation is therefore not present. In the control run (Fig. 4b), on the other hand, the transitions are much more frequent, at a rate of a dozen transitions per decade, and the coupled mode does arise. The degree of intermittency weakens even further with increasing atmospheric surface drag (Fig. 4c); since the two jet states become also less separated in 
(a)

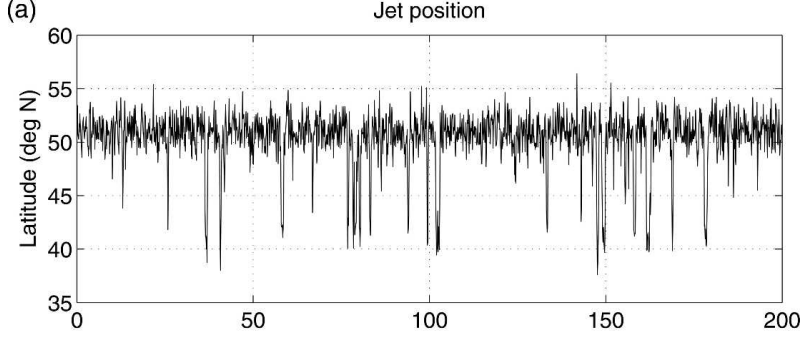

(b)

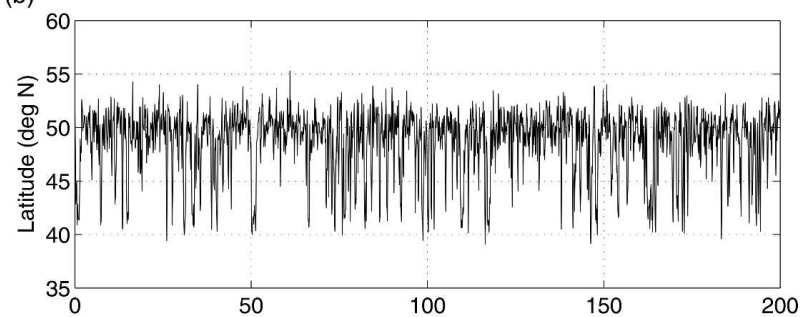

(c)

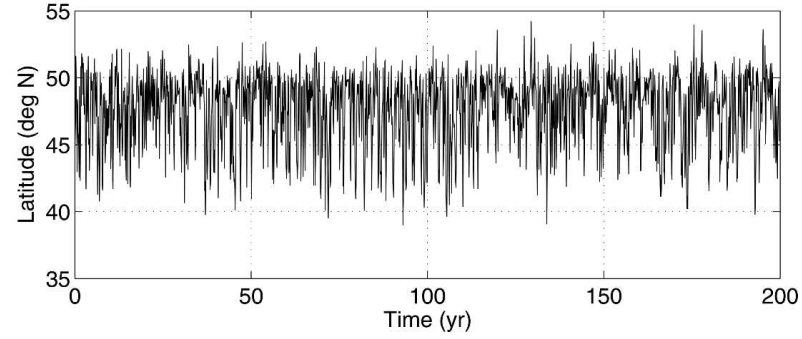

FIG. 4. Jet position time series (40-day averages): (a) experiment 2 (slow spindown); (b) experiment 1 (control run; intermediate spindown); and (c) experiment 3 (fast spindown).

latitude, the variability associated with the coupled mode accounts here for less of the model's total variance.

Thus, the nonlinear coupled oscillation occurs within a limited range of the atmospheric surface friction parameter. This range corresponds to the atmospheric circulation that is weakly bimodal and not too intermittent.

\section{b. Composite 9-yr cycle of the control run}

In Fig. 5a we show the composite cycle of the atmospheric jet position and ocean kinetic energy for the control run's coupled oscillation. The jet position time series leads that of ocean kinetic energy by roughly a quarter of a cycle. This cycle is asymmetric in the jet position, with a longer high-latitude stage and a shorter low-latitude stage, while the kinetic energy cycle is more symmetric.

\section{c. Sensitivity to ocean forcing}

To quantify nonlinear sensitivity of the atmospheric transitions to ocean-induced SST anomalies, we have
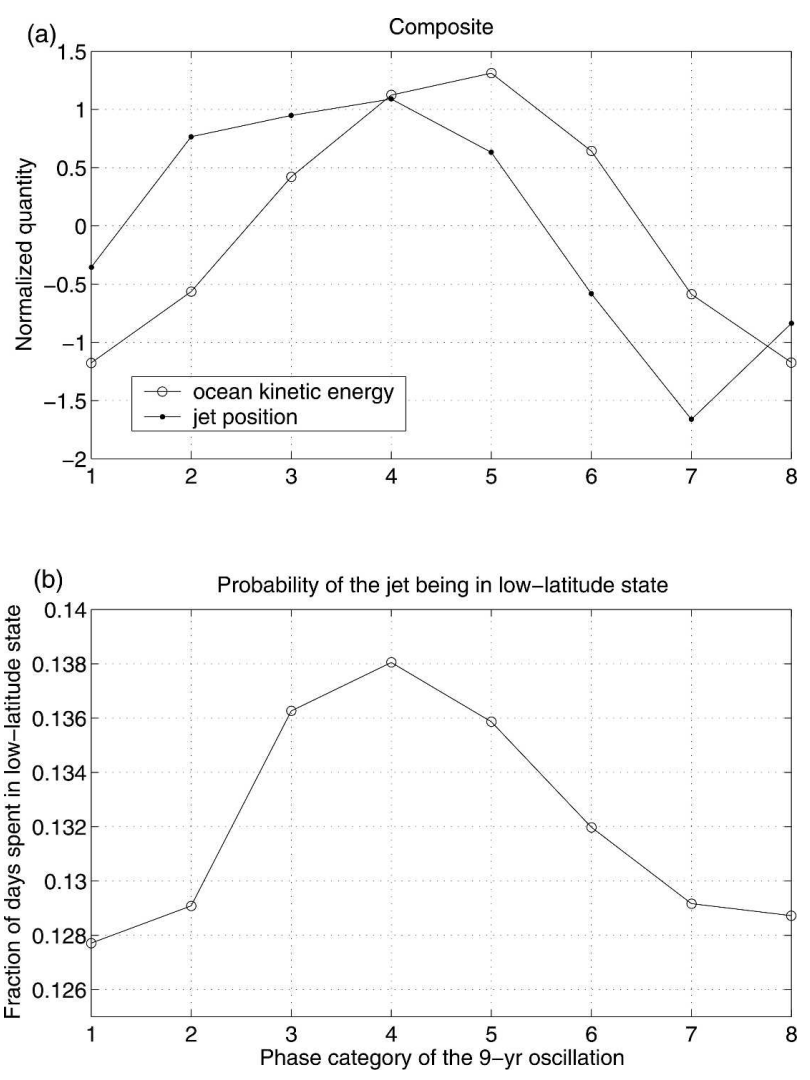

FIG. 5. Composites of characteristic features of the model, keyed to eight phase categories of the 9-yr coupled oscillation of experiment 1 (control run; intermediate spindown): (a) ocean kinetic energy and atmospheric jet position in the control run; and (b) conditional probability of the low-latitude jet position, given prescribed ocean states corresponding to the oscillation's phase categories (experiment 12).

performed long integrations of the atmosphere-oceanmixed layer component of our model, forced by ocean circulation composites (experiment 12 of Table 1). The composites were keyed to the eight phases of the 9-yr oscillation in the control run (experiment 1). We computed, in each experiment, the probability of the lowlatitude state, defined as the number of days spent in this regime divided by the total number of days in the time series. The results are shown in Fig. 5b.

The probability changes by about $10 \%$ of its mean value during the course of the oscillation; the highenergy ocean state (Fig. 5a) is more likely to induce atmospheric transition to the low-latitude regime and vice versa. Thus, the atmospheric high-latitude jet state favors eastward extension of the oceanic jet, and an overall more energetic ocean state (phases 2,3 , and 4 in Fig. 5a). This ocean state, in turn, favors more frequent atmospheric transitions to the low-latitude state (Fig. $5 b)$, and the negative swing of the coupled cycle begins (phases 5, 6, and 7 in Fig. 5a). 


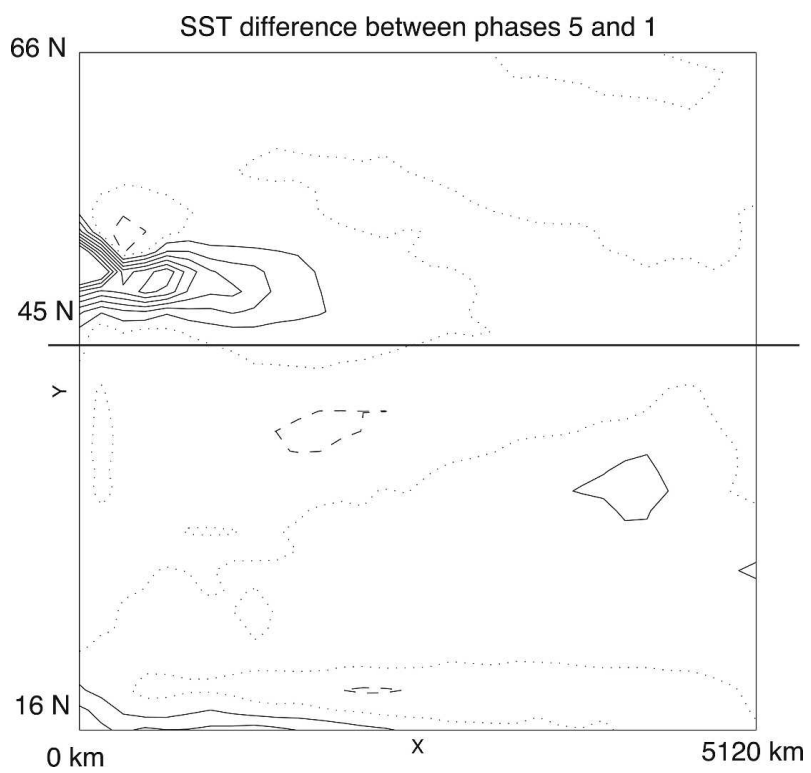

FIG. 6. Difference between composite SSTs from phases 5 and 1 of experiment 12 (see Fig. 5b). Contour interval is $0.5^{\circ} \mathrm{C}$, negative contours are dashed, zero contours are dotted.

We next examine the SST anomalies that result in the changes of low-latitude jet occurrence frequency, and plot in Fig. 6 the difference between the composite SST fields associated with phases 5 and 1 of the oscillation in experiment 12 (Figs. 5a,b). The increased probability of the atmospheric low-latitude state is thus induced by the elongated, monopolar pattern of positive SST anomalies centered roughly at the location of the climatological eastward jet extension in the ocean. Similarly, negative SST anomalies in the oceanic jet extension region induce atmospheric preference for less frequent transitions to the low-latitude atmospheric state (not shown).

Changes of $10 \%$ in the occurrence frequency of the low-latitude regime, like in Fig. 5b, as well as SST anomaly patterns similar to that in Fig. 6 can in fact be obtained in atmosphere-only simulations conditioned on the states of the coarse-resolution ocean model (not shown). If this is the case, then why does the coarseresolution ocean model, coupled to the same atmospheric component, not exhibit the coupled decadal variability? In section 4 we will show that the SSTforcing pattern in Fig. 6 in the higher-resolution ocean integration exhibits increased persistence due to more active ocean eddies; this increased persistence is, therefore, a primary reason for the existence of the coupled mode.

\section{Role of ocean nonlinearity}

In this section, we consider the following two questions: "what determines the time scale of the nonlinear

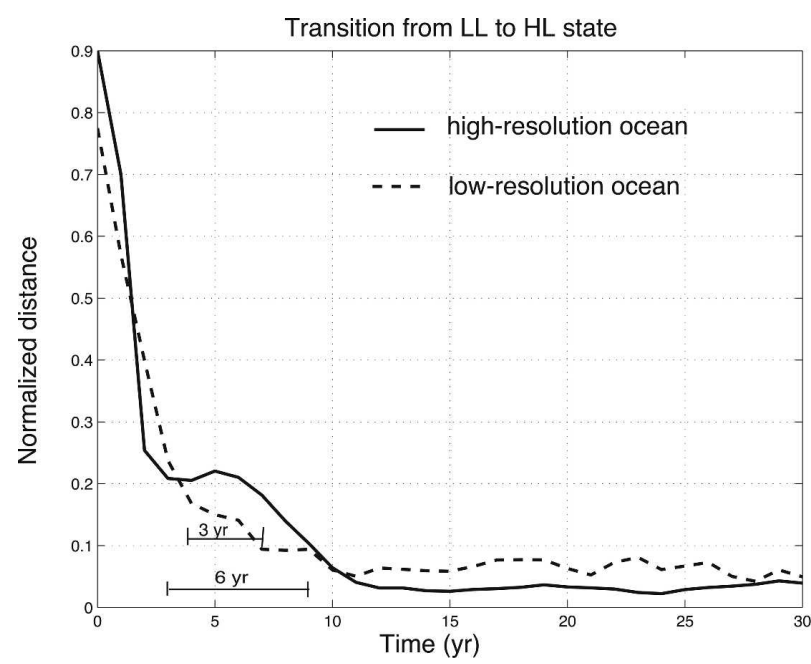

FIG. 7. Ocean adjustment experiments (experiments 13 and 14). Shown are rms distances from the final HL jet-forced ocean state, normalized by the distance between this latter state and the initial one, which is forced by the LL jet: experiment 13 (fine-resolution ocean; solid line) and experiment 14 (coarse-resolution ocean; dashed line). The distances shown are measured between the corresponding zonally averaged SST fields.

coupled oscillation?" and "how important are ocean eddies in forcing SST patterns that ensure effective ocean-atmosphere coupling?"

\section{a. Ocean adjustment to switches between atmospheric forcing regimes}

To test the hypothesis that the time scale of the coupled oscillation is set by nonlinear oceanic adjustment to switches between high- and low-latitude atmospheric forcing regimes, we perform ocean adjustment experiments: experiments 13-15 in Table 1 (see section 1b). The results from experiments 13 and 14 are plotted in Fig. 7 for the transition from the low- to the highlatitude forcing regime. Here we plot the root-meansquare (rms) distance (defined as a Euclidean distance between two vectors, i.e., the square root of the sum of squared differences between corresponding components of the two vectors) between the vector of the instantaneous zonally averaged SST field and the vector of the zonally averaged SST climatology forced by the atmospheric high-latitude jet.

Prior to the analysis, we have applied a 5-yr runningmean filter to all the fields considered. The results depend very little on the filter's window size: they are qualitatively the same if 3- or 7-yr filters are used instead (not shown). The distance plotted is normalized by the distance between the time-averaged high- and low-latitude states. Solid lines show the results from experiment 13, which uses the fine-resolution ocean, 
and light lines show the results from experiment 14 with coarser ocean resolution. In both cases, the distance decreases fairly rapidly over about 3-5 yr, from values of about $0.8-0.9$ to values below 0.2 . The asymptotic values below 0.1 are reached after about $10 \mathrm{yr}$ and differ from zero due to the presence of intrinsic ocean variability in the model.

The initial, fast stage of the adjustment may be called the "advective stage," since it is characterized by the northward migration of the point at which the western boundary current separates from the boundary, in response to the shift in the atmospheric jet position. This northward migration can be seen in Figs. 8a,c for the fine-resolution case (experiment 13) and in Figs. 8b,d for the coarse-resolution case (experiment 14). The duration of this stage is set by the ocean advective time scale: the distance between the two separation points, before and after the migration, divided by the characteristic velocity of the western boundary current equals approximately 2-3 yr in experiment 13 and 3-5 yr in experiment 14 .

The following, slower stage of adjustment may be called the "eddy-driven stage"; see Figs. 8e,g and Figs. 8f,h for the high- and coarse-resolution cases, respectively. This stage takes another 3-7 yr and is governed by eddy dynamics (Dewar 2003). In the fine-resolution case, the rapid adjustment stage (Figs. 8a,c,e) results in relocation of the separated oceanic jet to a new latitudinal position, while the slow stage (cf. Figs. 8e,g) is characterized primarily by the increasing intensity of the jet and the degree of eastward penetration into the ocean basin. In contrast, large-amplitude circulation anomalies in the low-resolution case (Figs. 8b,d,f,h) are localized closer to the western boundary, with lesser penetration of the jet into the basin's interior.

A major difference between the oceanic adjustment process in fine- and low-resolution ocean models is a slower eddy-driven stage of SST adjustment; the corresponding time scale is of about 3-4 yr for the coarseresolution model and 6-7 yr for the fine-resolution model. This difference of about $2-3 \mathrm{yr}$, as well as different patterns of transient SST anomalies (see below), is responsible for the coarse-resolution integration's not exhibiting the coupled decadal oscillation characteristic of the fine-resolution case (Table 1).

The evolution of SST anomalies during the adjustment is plotted in Fig. 9. Figures 9a,b,d,e,g,h present results for the fine- (experiment 13) and low-resolution (experiment 14) version of the ocean model, while Figs. $9 \mathrm{c}, \mathrm{f}, \mathrm{i}$ plots the evolution of zonally averaged SST anomalies for both simulations. The anomalies shown are computed with respect to the final state, which is also characterized by a high-latitude mean position of the oceanic jet and approximately reached by year 10 of either simulation (Figs. 9g-i).

The difference between the fine- and coarseresolution ocean simulations is most clearly seen in comparing the SST anomalies at year 4 (Figs. 9d-f). The fine-resolution model is characterized here by a tongue of positive SST anomalies along the new, highlatitude location of the separated jet (Fig. 9d), while the low-resolution case's SST anomalies in this region are weaker and have a smaller east-west scale. This results in a pattern of zonally averaged SST anomalies that differs considerably from one model version to the other (Fig. 9f). The SST anomalies in Fig. 9d are quite similar to those in Fig. 6 and thus tend to force more frequent transitions of the atmospheric jet to the lowlatitude state. The increased persistence of these anomalies is quite clearly associated with ocean eddy dynamics, since they do not arise in the weaker eddy, coarse-resolution ocean experiments.

The SST anomalies in Fig. 9d are associated with the "overshoot" of the ocean jet after the fast, advective stage of the adjustment: the latitude of the eastward jet at year 4 (Fig. 8e) is to the north of the ocean jet's final latitude (Fig. 8g); the latter latitude coincides with the position of the atmospheric jet's high-latitude state. Thus, the positive tongue of SST anomalies to the north of the high-latitude atmospheric jet induces transitions to the low-latitude atmospheric state; conversely, negative SST anomalies to the south of the low-latitude atmospheric jet (not shown) favor the high-latitude state of this jet. The transient, but fairly persistent overshoot of the oceanic jet that determines these SST anomalies is maintained by the oceanic baroclinic eddies via a nonlinear rectification process. According to Berloff (2005), these eddies act on the large-scale oceanic flow as a small-scale stochastic forcing; this forcing, though, is organized by the combined action of the nonlinearity and the $\beta$ effect so as to preferentially deposit positive potential vorticity anomalies to the north of the jet, and negative anomalies to the south. In the coupled integrations using the coarse-resolution ocean model, the eddy field is weak, and the eddy-driven stage of the adjustment is both shorter and less effective; consequently, the coupled decadal mode is not found in these experiments.

We conclude, therefore, that ocean eddy dynamics is essential for the coupled oscillation in setting up SST anomalies that are able to affect the atmospheric flow in a way that maintains and reinforces the coupled mode. They are also instrumental in setting up the time scale of the oscillation. The latter time scale is related to the duration of the eddy-driven adjustment stage, which determines how long SST anomalies in the vicin- 
$66 \mathrm{~N}$

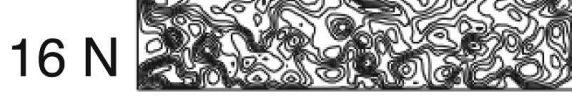

(a)

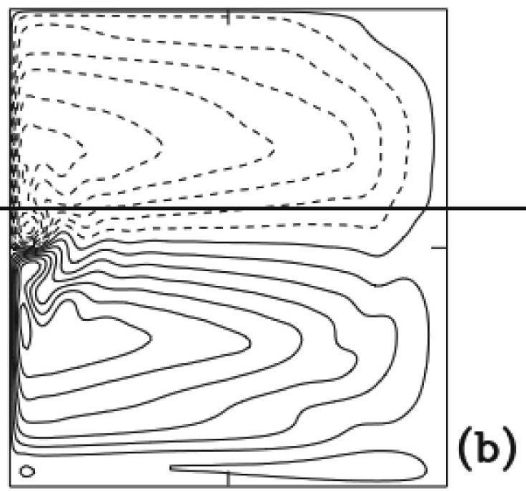

$66 \mathrm{~N}$

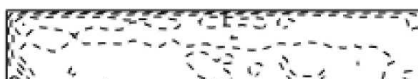

$45 \mathrm{~N}$

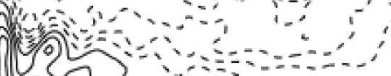

.

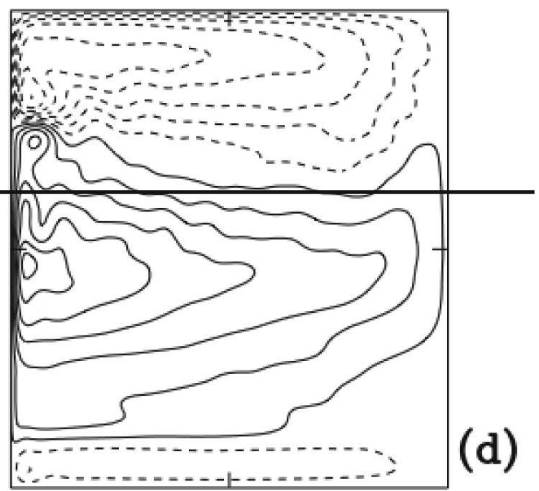

$16 \mathrm{~N}$
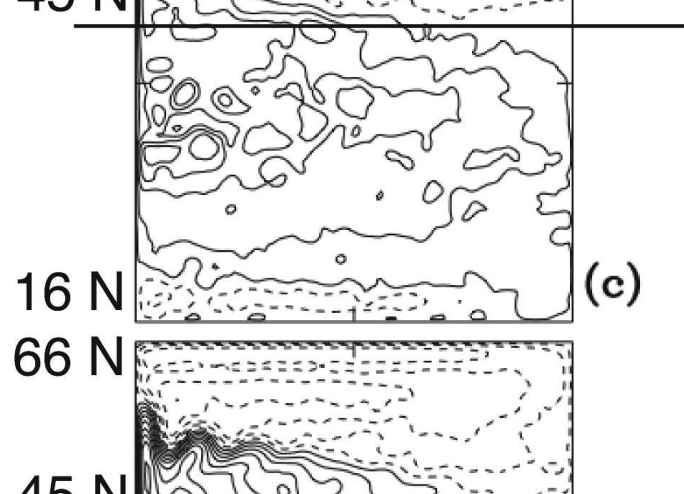

$45 \mathrm{~N}$
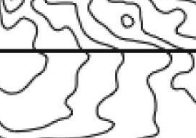

रेट
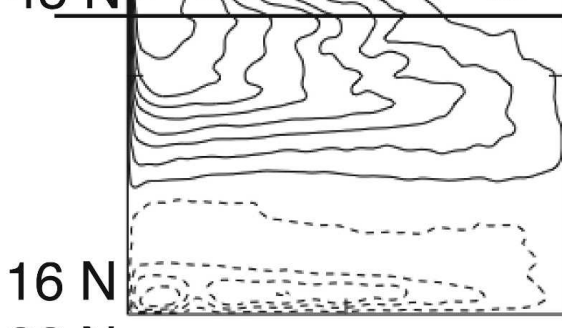

.

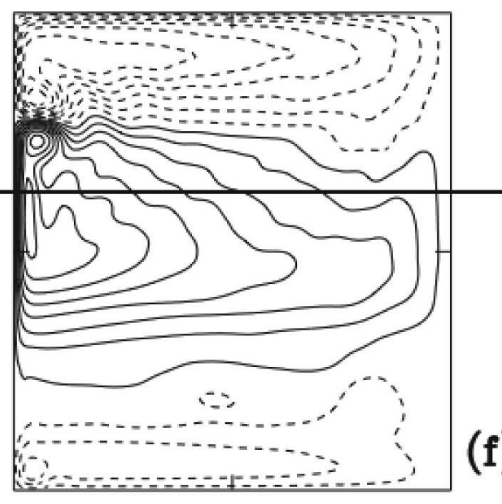

(f)

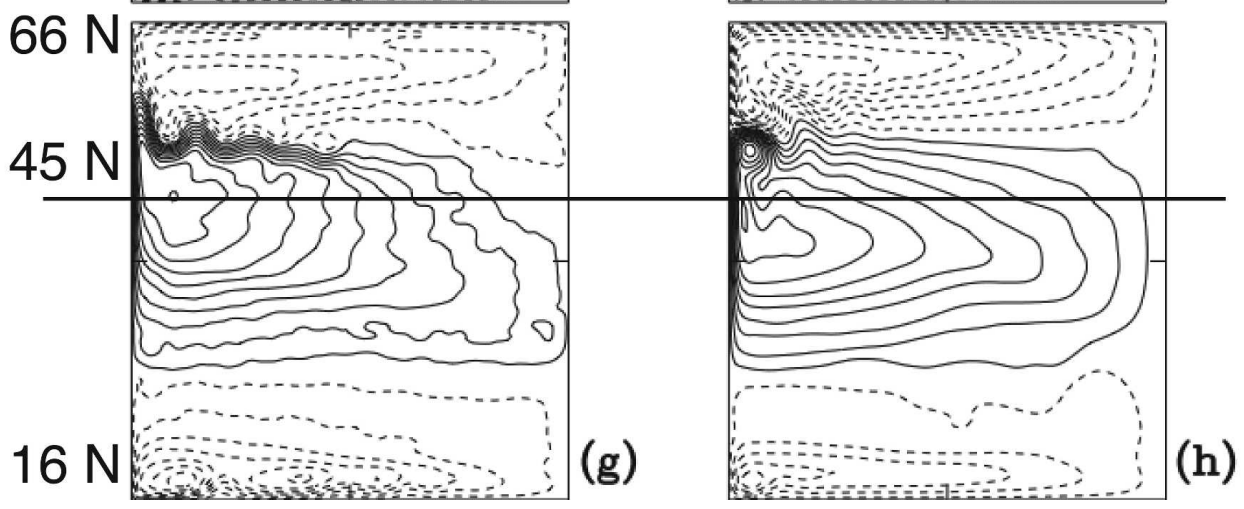

FIG. 8. Snapshots of the upper-layer streamfunction $\Psi_{1}$ for the ocean adjustment experiments in Fig. 7: (a), (c), (e), (g) experiment 13 (adjustment of the fine-resolution ocean) and (b), (d), (f), (h) experiment 14 (adjustment of the coarse-resolution ocean). (a), (b) Initial (unfiltered) state; (c), (d) the state after $2 \mathrm{yr}$; (e), (f) after $4 \mathrm{yr}$; and (g), (h) after $10 \mathrm{yr}$. The contour interval is $5 \mathrm{~Sv}\left(1 \mathrm{~Sv} \equiv 10^{6} \mathrm{~m}^{3} \mathrm{~s}^{-1}\right)$; negative contours are dashed. 

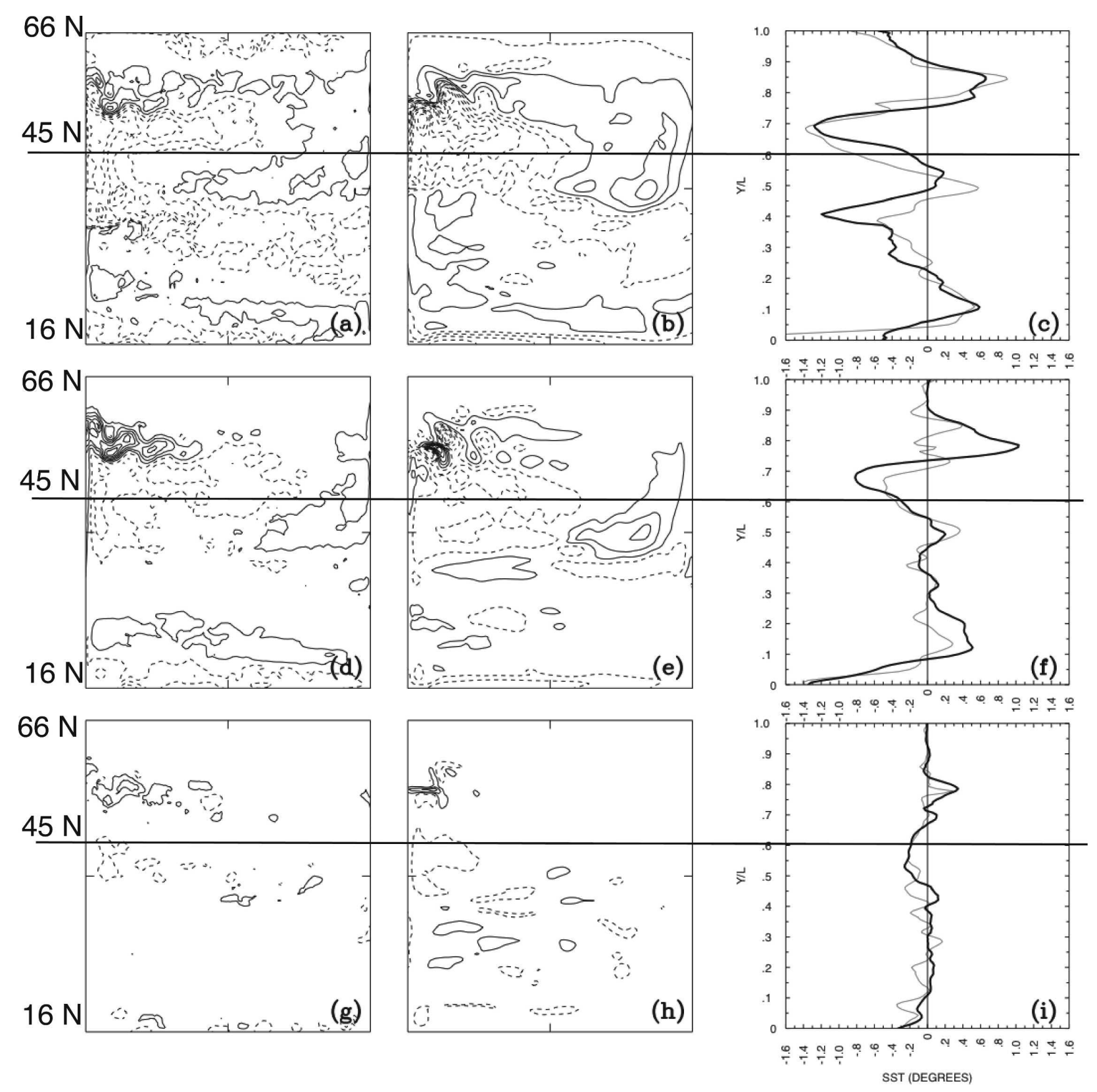

FIG. 9. Snapshots of SST anomalies (final state has zero anomaly) for the experiments of Fig. 7: (a), (d), (g) experiment 13 (adjustment of the fine-resolution ocean); (b), (e), (h) experiment 14 (adjustment of the coarse-resolution ocean); the contour interval is $5^{\circ} \mathrm{C}$, negative contours are dashed. (c), (f), (i) Zonally averaged SST for experiment 13 (heavy solid line) and experiment 14 (light solid line). (a)-(c) The state after 2 yr; (d)-(f) after 4 yr; and (g)-(i) after $10 \mathrm{yr}$.

ity of the eastward jet can exist in the absence of local atmospheric forcing; the resulting multiyear time lag leads to the decadal-interdecadal oscillation. To further study the role of eddies in setting up the time scale of the oscillation, we next consider experiments that use a lower ocean bottom drag coefficient, namely, experiments 7 and 15 in Table 1.

\section{b. Coupled experiment with low ocean bottom drag}

\section{1) Climatology, spectra, And composite CYCLE}

The major difference between climatological patterns of ocean circulation from the coupled run with low ocean bottom drag and their analog for the control 
run, which uses a larger value for the bottom drag, is in the magnitude and spatial extent of the inertial recirculations. The IR are stronger and occupy a larger area in the low-drag experiment (not shown). In this regard, the results for the low-drag experiment are thus more realistic.

The spectrum of annually averaged data from experiment 7 (not shown) exhibits a broad spectral peak centered at about a 20 -yr period, in both jet position and ocean kinetic energy time series. The oceanic spectrum also exhibits a 5-yr peak representative of a coupled Rossby wave signal (see section 5). The 20-yr oscillation has atmospheric and oceanic spatiotemporal patterns (not shown) that resemble those of the 9-yr coupled oscillation discussed in K06.

The composite cycle of the 20-yr oscillation in jet position and ocean kinetic energy (not shown) is also very similar to that of the 9-yr oscillation of the control coupled run. In particular, these two scalar quantities exhibit the same phase relations as the 9-yr coupled oscillation of the control run (Fig. 5a): the jet position time series leads that of ocean kinetic energy by a quarter of a cycle.

To summarize, the low bottom drag run thus exhibits the same type of oscillation as the control run, but with a period that is roughly twice as long.

\section{2) Adjustment EXPERIMENTS}

To better understand this difference in period, we now compare ocean adjustment experiments that use high and low values of the ocean drag (experiments 13 and 15 in Table 1). The results are shown in Fig. 10 for the adjustment of the upper-layer streamfunction $\Psi_{1}$ to a switch from atmospheric forcing by a low-latitude (LL) jet to forcing by a high-latitude (HL) jet state. The rapid, advective phase of the adjustment (see section 4a) has the same time scale in the two runs, while its subsequent, eddy-driven component is considerably longer in the run with low ocean drag: 15 versus 6 yr. The eddy-driven adjustment time scale has, therefore, a decisive effect on the period of the coupled oscillation.

In the eddy-driven adjustment, the lower layers' potential vorticity fields adjust to the forcing of the upper layers. The time scale of this adjustment via eddy fluxes of potential vorticity (Dewar 2003) is longer in the low bottom drag run, since lateral eddy fluxes have to do most of the dissipation in the deepest layer, as opposed to direct dissipation via bottom drag in the control run.

\section{Coupled Rossby wave mode}

We now turn to the coupled Rossby wave mode, which is present in all the coupled experiments (see

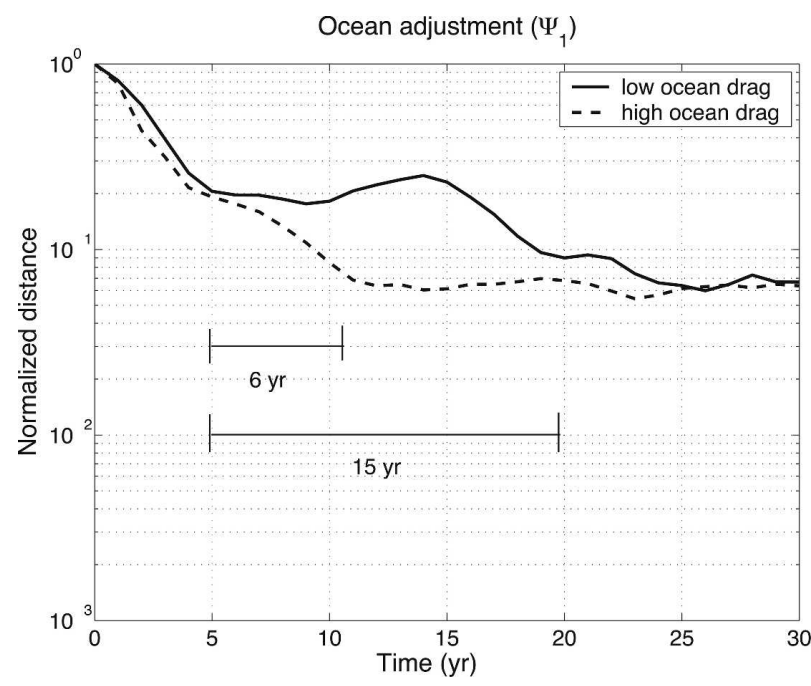

FIG. 10. Comparison of ocean adjustment time scales of $\Psi_{1}$ between experiments 13 (adjustment of the fine-resolution, high bottom drag ocean; dashed line) and 15 (adjustment of the fineresolution, low bottom drag ocean; solid line).

Table 1) and has a period of 3-5 yr [see sections $3 \mathrm{a}$ and $4 \mathrm{~b}(2)$ ]. To visualize this mode, we regress oceanic and atmospheric fields onto $1-10$-yr bandpass-filtered ocean kinetic energy from experiment 3, multiplied by -1 , and normalized to have unit variance.

The results for atmospheric temperature are plotted in Fig. 11. The evolution is characterized by a westward-propagating wave-4. Circulation anomalies associated with this signal in the upper-ocean layer are shown in Fig. 12. They have the same spatial scale as that of the atmospheric wave (Fig. 11) and also propagate westward. SST evolution is depicted in Fig. 13. Note that the SST wave is in phase with atmospheric temperature and in quadrature with $\Psi_{1}$. Moreover, SSTs and atmospheric temperature anomalies have a similar magnitude, with SST anomalies being slightly larger, which indicates that SST anomalies force atmospheric response rather than vice versa.

It follows that this mode is a coupled Rossby wave of the type studied by Goodman and Marshall (1999) in a linear channel model of both atmosphere and ocean. Our model has a closed ocean basin, and the period of this linear oscillation is equal to the time it takes for the lowest-mode Rossby wave of this basin to cross it (Sheremet et al. 1997; Chang et al. 2001). The dominant atmospheric wave associated with this Rossby basin mode is equivalent barotropic and its wavenumber 4 is also set by the ocean basin's extent, which is equal to $1 / 4$ of the channel length. This wave does exist in uncoupled atmospheric experiments (Kravtsov et al. 2005a), but exhibits no regularity in the interannual 

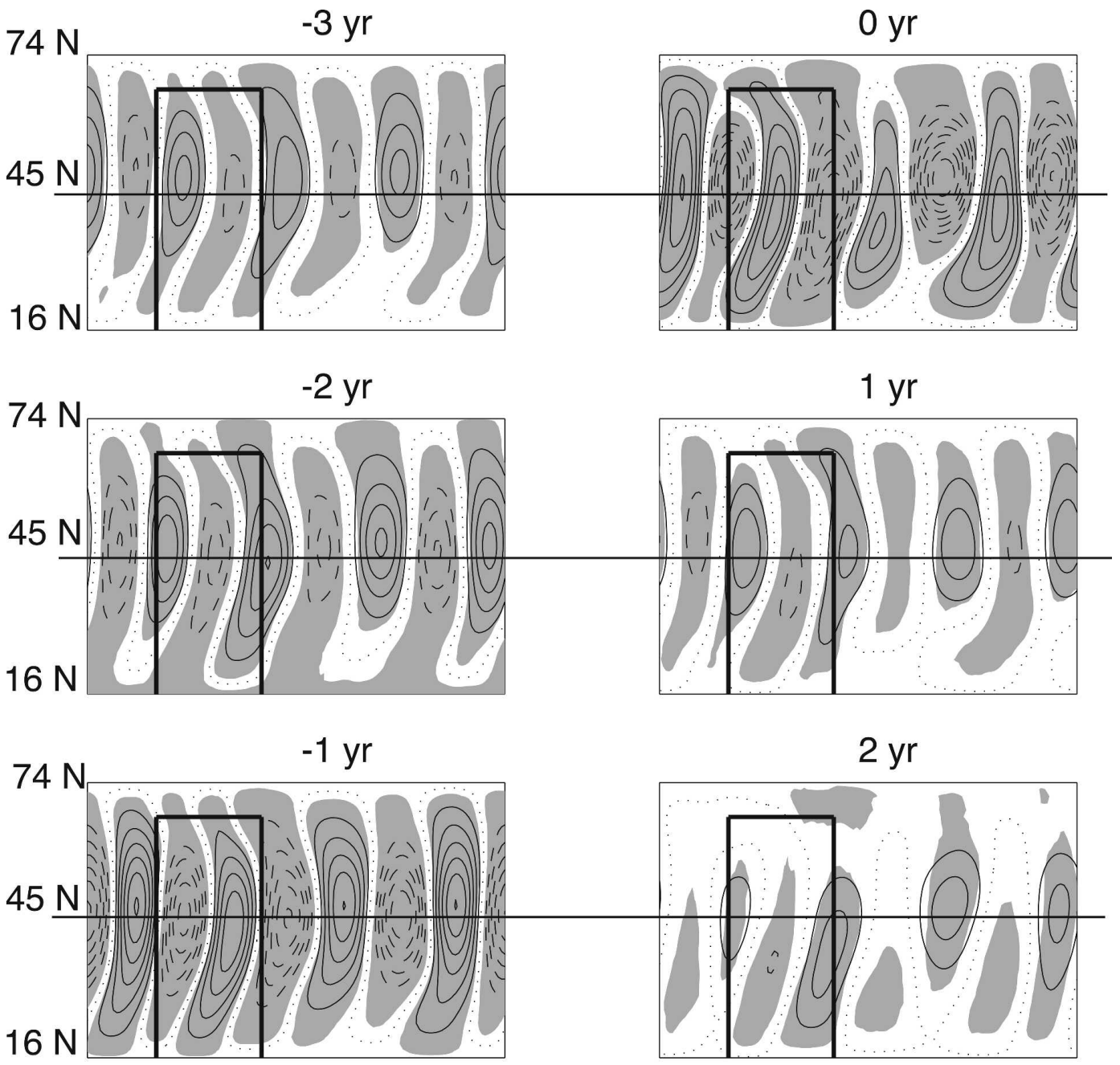

\section{Atmospheric temperature $(\mathrm{Cl}=0.1$ degrees $\mathrm{C})$}

FIG. 11. Evolution of atmospheric temperature fields during the coupled Rossby wave cycle: lagged regression of atmospheric temperature onto centered normalized 1-10-yr bandpass-filtered time series of ocean kinetic energy (experiment 3; fast atmospheric spindown), multiplied by -1 . Units and contour interval are given in the figure legend, the lag value is given in the heading of each panel. Lag 0 corresponds to negative kinetic energy anomaly, while positive lags correspond to $E_{k}$ time series leading the atmospheric temperature evolution. Negative contours are dashed, the zero contour is dotted. Shading denotes values statistically significant at the $5 \%$ level with respect to a linear stochastic process null hypothesis. Geometry is not to scale.

band. In the coupled integration, it is amplified via a positive feedback with the ocean component's Rossby basin mode, as described by Goodman and Marshall (1999) in their channel model.

\section{Concluding remarks}

\section{a. Summary}

This paper analyzes in greater detail a novel, highly nonlinear mechanism of coupled ocean-atmosphere behavior in the midlatitudes. This mechanism depends crucially on the nonlinear dynamics of both fluids, namely (i) nonlinear sensitivity of atmospheric flow to ocean-induced SST anomalies, which involves changes in the frequency of occurrence of two distinct zonalmean zonal flow regimes; and (ii) ocean eddy dynamics of the so-called inertial recirculations (IRs), which enhance the SST anomalies that affect the atmosphere, as well as determine the time scale of the coupled signal. The latter signal appears in the spectral analysis of both the atmospheric and oceanic model time series as a 


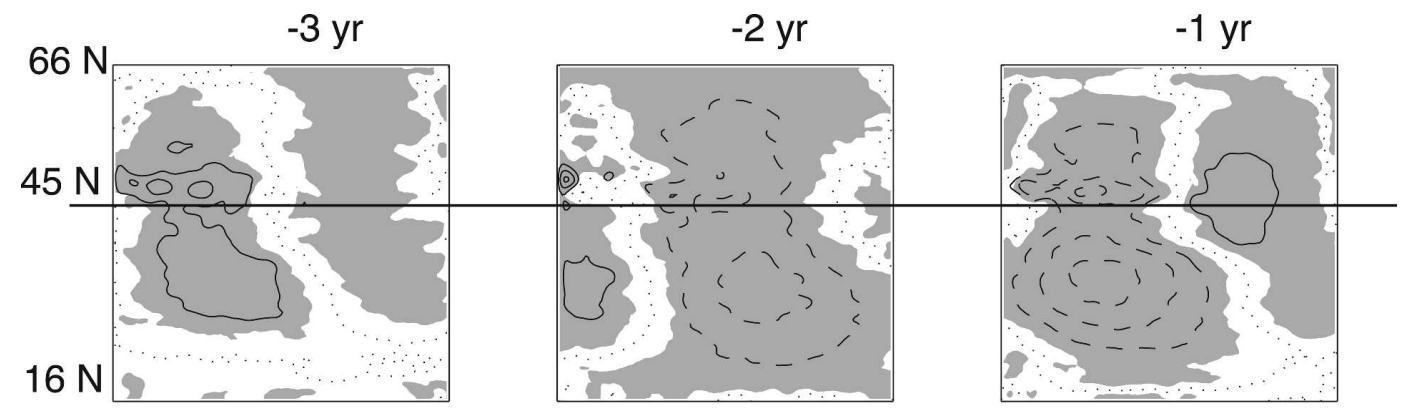

$\Psi_{1}(\mathrm{Cl}=1.2 \mathrm{~Sv})$

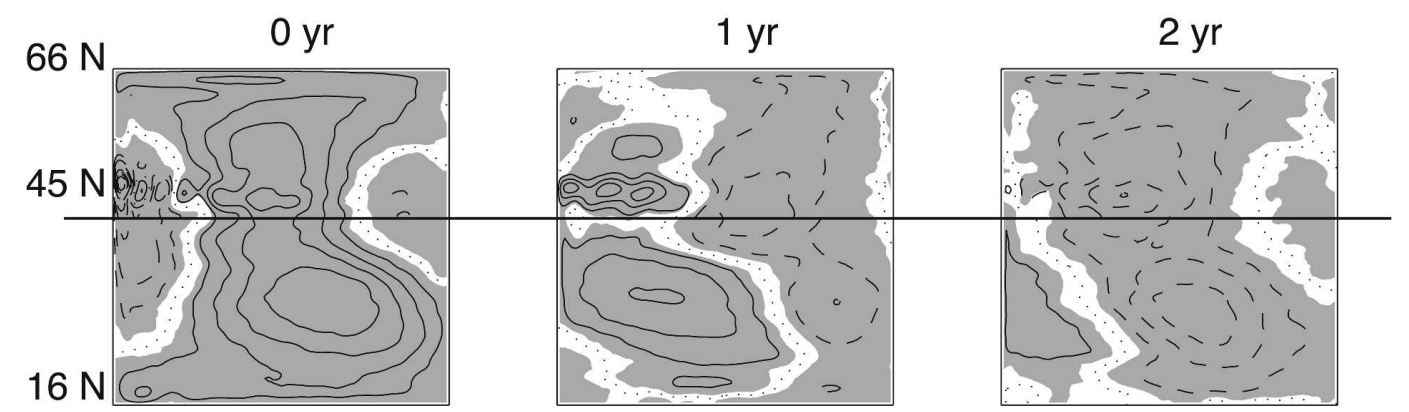

FIG. 12. Same as in Fig. 11, but for the ocean's upper-layer streamfunction $\Psi_{1}$.

broad peak in a decadal range (Fig. 1). This peak is absent from all uncoupled integrations, as well as from fully coupled integrations that use a coarser-resolution ocean model, with less eddy activity (see Table 1 ).

In section 3, we have discussed the role of atmospheric nonlinearity. The nature of atmospheric behavior (i.e., bimodal versus unimodal) depends on the value of the surface friction coefficient. The bimodality is present for intermediate and low values of the surface drag, while the behavior at high drag is unimodal (see Kravtsov et al. 2005a; Fig. 2 herein). Coupled decadal oscillations appear only in the cases in which bimodality is present; see Figs. $1 \mathrm{~b}$ and $3 \mathrm{~b}$. The variance associated with this coupled oscillation decreases as one tracks the model's behavior to higher values of surface drag. The atmospheric signal in this band appears as a decadal modulation of the transitions from the dominant, high-latitude jet state to the much rarer low-latitude jet state.

At very low values of surface friction, however, while the bimodality is very pronounced (Fig. 2a), the coupled signal is not found either (Fig. 3a), just like in the cases with high-drag, unimodal behavior. This has to do with the transitions from the atmosphere's preferred high-latitude state to its low-latitude state becoming too infrequent, at a rate of about one transition per decade, for low surface friction; in this case, the ocean cannot influence their occurrence frequency on decadal time scales (Fig. 4).

We have chosen the westerly jet position to characterize the state of the atmosphere during the coupled decadal-interdecadal cycle and the kinetic energy to characterize that of the ocean. The composite decadal cycle of these two quantities, both of which are highly relevant to the coupled variability, shows that the jet position leads ocean kinetic energy by a quarter of a cycle (Fig. 5). Given a high-latitude jet, the ensuing high-energy state of the ocean is associated with the development of an intense eastward jet, which penetrates far into the ocean basin, and vigorous IRs. The resulting oceanic circulation anomalies cause SST anomalies (Fig. 6), which tend to induce more frequent transitions of the atmosphere to its low-latitude state. These repeated transitions result in the collapse of the oceanic jet and development of SST anomalies that favor the return of the atmosphere to its high-latitude state (Fig. 5b).

Section 4 provided evidence for the role of ocean eddy dynamics in the coupled oscillation. In particular, we studied oceanic adjustment to a sudden switch in the atmospheric state from a low- to a high-latitude jet state, and vice versa, and we used three different models (experiments 13-15 in Table 1): the fine-resolution ocean model of the control run, an ocean model version 


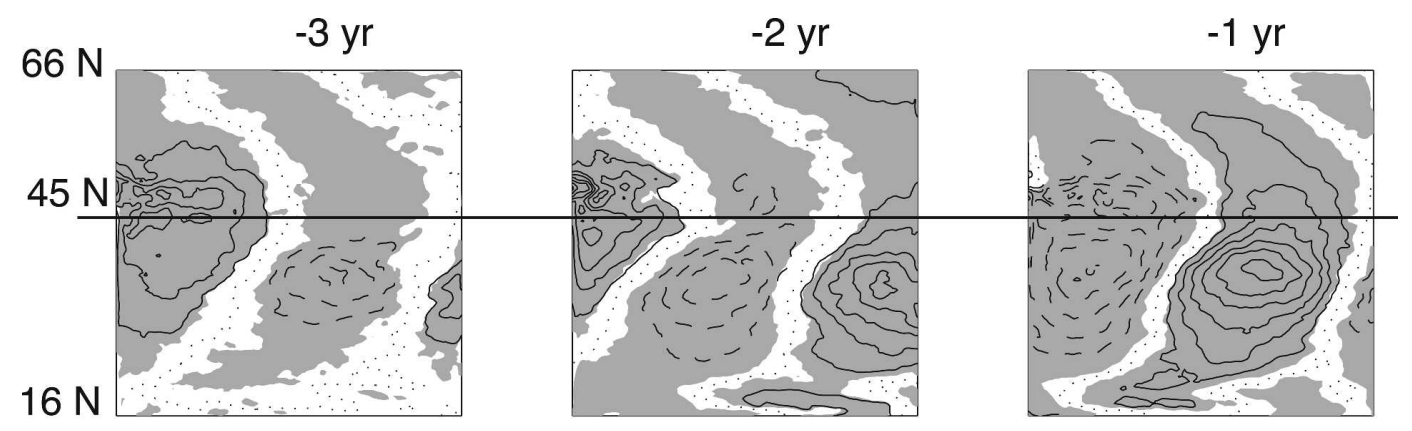

\section{$\operatorname{SST}(\mathrm{Cl}=0.1$ degrees $\mathrm{C})$}

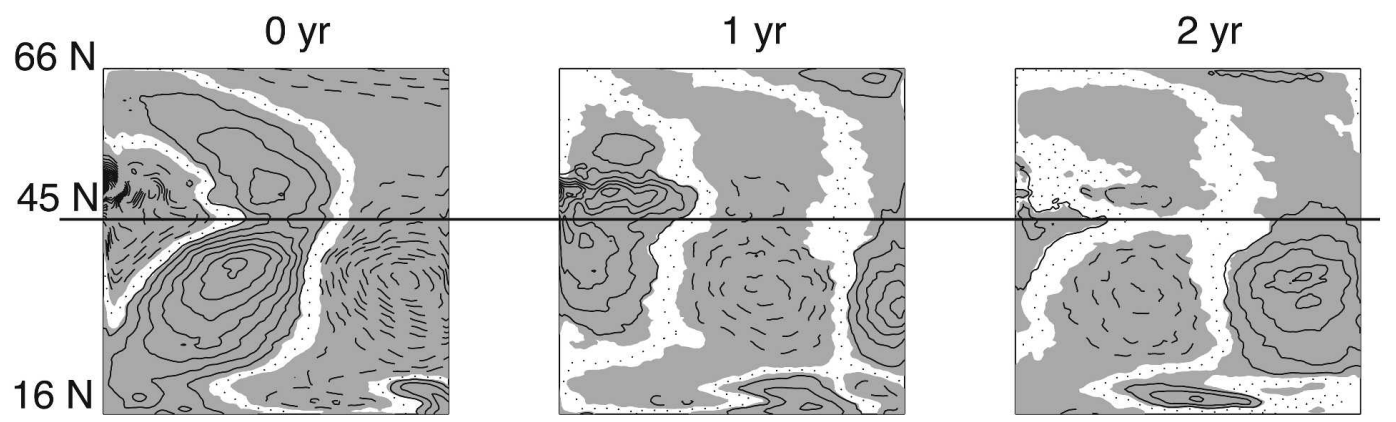

FIG. 13. Same as in Fig. 11, but for SST.

that has a coarser resolution and hence a much weaker eddy activity, and a fine-resolution ocean model version with a much smaller value of the ocean bottom drag coefficient than in the control run. In the coupled experiments that use these three models, only those with the fine-resolution ocean exhibit a coupled oscillatory mode. The period of this mode is roughly twice as long in the run with low ocean bottom drag, while it is entirely absent from the runs with a coarse-resolution ocean (experiments 1, 4, and 7 in Table 1).

We found that the ocean's adjustment to a predominantly high- or low-latitude jet forcing has two components: a fast, advective phase and a slower, eddy dynamics-dominated phase (Figs. 7-9). The duration of the former phase is of 3-5 $\mathrm{yr}$ and depends relatively little on the ocean bottom drag (Fig. 10), while the latter phase can more than double in length (from 6 to $15 \mathrm{yr}$ ) as the bottom drag is reduced.

Higher resolution of the ocean model, and the resulting higher eddy activity, also give rise to more persistent anomalies in the separated ocean jet region (Figs. 8 and 9). The spatial pattern of these anomalies produces heat flux forcing on the atmosphere that favors atmospheric return to the initial, preadjustment state (cf. Figs. 9d and 6). We conclude, therefore, that IR anomalies force the SST anomalies, which are able to efficiently affect atmospheric transitions between the two distinct regimes.

In addition to the nonlinear, 10-20-yr coupled mode described above, we have also found a coupled mode that depends neither on atmospheric bimodality, nor on the ocean's eddy dynamics. This interannual, linear coupled Rossby mode has been discussed in section 5 . Phase relations between the oceanic and atmospheric fields (Figs. 11-13) show that this mode is analogous to the one studied by Goodman and Marshall (1999) in a channel geometry. Given our model's closed ocean basin, the time scale of this oscillation appears to be set by the time it takes to the oceanic Rossby wave to cross the basin.

We have argued that ocean eddies contribute substantially to SST anomalies that play a key role in the coupled oscillation, as well as to the oceanic adjustment time scale and, therewith, to the oscillation's period. We have done so in the present paper only by comparing the results of different coupled and uncoupled integrations. To understand in greater depth and detail the way ocean eddies operate in the coupled context, we have developed a novel technique to parameterize ocean eddies stochastically in a coupled model that uses a coarser-resolution ocean. We have shown, in particular, that the coupled behavior absent from a model that 
uses a coarse-resolution ocean component is recovered when our novel stochastic eddy parameterization is applied (Berloff et al. 2007).

\section{b. Discussion}

Our model is highly idealized (low vertical resolution, quasigeostrophic dynamics, idealized geography, simplistic parameterizations of heat and momentum exchange between the ocean and the atmosphere, etc.) and therefore its results (likewise, the results from any type of an idealized model or theory) must be treated with caution when relating them to the real world. Having said that, we will now argue for a broad correspondence between the patterns and time scales of variability we have modeled and the observed ones.

The highly nonlinear coupled mode discussed here does not arise from an intrinsic oceanic mode; it involves, instead, a coupled adjustment process, in which the ocean modifies intrinsic atmospheric variability on decadal-interdecadal time scales via a nonlinear feedback due to interaction between the ocean's large-scale flow and turbulent eddies. The most realistic results, in terms of the spatial extent and strength of the oceanic inertial recirculations, were obtained for low values of the oceanic bottom drag. For the optimal values of the bottom drag and resolution in the ocean model, the period of our nonlinear coupled mode is of about 15-25 yr, rather than the $9-10 \mathrm{yr}$ for the control run. This interdecadal band has been found to be prominent in climatic signals over the North Atlantic (Plaut et al. 1995; Moron et al. 1998), as well as over the North Pacific (Chao et al. 2000) and in global SSTs and surface air temperatures (Folland et al. 1984; Ghil and Vautard 1991).

An interannual mode of intrinsic oceanic variability, called the gyre mode, has been discovered and well documented in a hierarchy of increasingly realistic ocean-only models (Jiang et al. 1995; Speich et al. 1995; Ghil et al. 2002b; Simonnet et al. 2003a,b, 2006; Dijkstra and Ghil 2005). The gyre mode's fundamental period depends mainly on the nonlinear dynamics of the recirculation dipole near the separation of western boundary currents and is fairly independent of basin size. This period lies in the 5-10-yr band and seems to provide a plausible explanation for the 7-8-yr peak in North Atlantic SSTs (Moron et al. 1998), and sea level pressure (Da Costa and de Verdière 2002), as well as in the meridional displacements of the Gulf Stream axis (Dijkstra and Ghil 2005; Simonnet et al. 2005), the North Atlantic Oscillation index (Wunsch 1999), and the 335-yr-long time series of central U.K. temperatures (Plaut et al. 1995).

The spatial patterns of this mode bear certain simi- larities to those of our highly nonlinear coupled variability: they both involve modulations of the oceanic eastward jet's meridional position, intensity, and eastward penetration into the basin, as well as associated reorganizations of the IR region. Still, Moron et al. (1998) show substantial differences between the basinwide SST patterns of the 13-14-yr mode (their Fig. 9) and the 7-8-yr mode (their Fig. 10) in the North Atlantic, although both have particularly strong anomalies along the east coast of North America, between the Florida Straits and the Great Banks. There is a good likelihood, therefore, that the present 15-25-yr coupled mode might contribute to the interdecadal climate variability documented not only in the North Atlantic (Deser and Blackmon 1993; Kushnir 1994; Moron et al. 1998), but also in the North Pacific (Mantua et al. 1997; Chao et al. 2000) and globally (Folland et al. 1984; Ghil and Vautard 1991).

That being said, it appears fortunate that our coupled model does not support the gyre mode; indeed, this fact allowed us to identify and study in some depth the novel, highly nonlinear, truly coupled mode, with its 15-25-yr period, described in the present study. The reasons behind the absence of the gyre mode in our ocean model require further investigation.

To make matters even more complicated and interesting, Hogg et al. $(2005,2006)$ have obtained a $15-y r$ oscillation in a coupled model similar to ours, but in which atmospheric behavior is not bimodal. Their oscillatory mode seems to be driven by intrinsic oceanic variability, whose spatial patterns resemble the gyre mode. In the work of Hogg et al. (2005, 2006), like in that of Feliks et al. (2004; see also Y. Feliks et al. 2005, personal communication), the intrinsic oceanic variability modulates the intrinsic modes of atmospheric variability on interdecadal or interannual time scales, respectively. Simonnet (2005) has shown that the gyre mode, in the presence of bottom friction, can be "quantized," according to basin size, that is it can exhibit harmonics depending on the number of eastward jet meanders accommodated by the basin. It is possible, therefore, to have gyre modes with both 7-8- and 15-yr periods.

We are thus faced with an embarrassment of riches: two or three different sources of midlatitude climate variability, either ocean driven or truly coupled. To distinguish between these types of midlatitude climate variability in observations and general circulation model simulations is not that easy, since the spatial patterns, dynamical mechanisms, and time scales of the associated modes bear certain similarities. A possible direction to follow in this regard is to look for the statistical signatures of ocean-atmosphere covariability 
that will differ from one mode to another. Such statistical studies will have to be complemented by a better understanding of the way the modes differ dynamically from each other. Provided statistical and dynamical insights that uniquely identify each of the modes are available, it will be possible to make inferences about which of the modes, or combinations thereof, contribute (if at all) to which frequency band of climate variability.

Acknowledgments. It is with pleasure that we thank D. Kondrashov and A. W. Robertson for helpful discussions. The comments of anonymous reviewers also helped to improve the presentation. This research was supported by NSF Grant OCE-02-221066 (all coauthors) and DOE Grant DE-FG-03-01ER63260 (MG and SK).

\section{REFERENCES}

Allen, R. M., and L. A. Smith, 1996: Monte Carlo SSA: Detecting irregular oscillations in the presence of colored noise. J. Climate, 9, 3373-3404.

Berloff, P., 2005: On rectification of randomly forced flows. $J$. Mar. Res., 31, 497-527.

— W. K. Dewar, S. Kravtsov, and J. C. McWilliams, 2007: Ocean eddy dynamics in a coupled ocean-atmosphere model. J. Phys. Oceanogr., in press.

Chang, K.-I., M. Ghil, K. Ide, and C.-C. A. Lai, 2001: Transition to aperiodic variability in a wind-driven double-gyre circulation model. J. Phys. Oceanogr., 31, 1260-1286.

Chao, Y., M. Ghil, and J. C. McWilliams, 2000: Pacific interdecadal variability in this century's sea surface temperatures. Geophys. Res. Lett., 27, 2261-2264.

Da Costa, E. D., and A. C. de Verdière, 2002: The 7.7-year North Atlantic oscillation. Quart. J. Roy. Meteor. Soc., 128A, 797817.

Deser, C., and M. L. Blackmon, 1993: Surface climate variations over the North Atlantic Ocean during winter: 1900-1989. J. Climate, 6, 1743-1753.

Dettinger, M. D., M. Ghil, C. M. Strong, W. Weibel, and P. Yiou, 1995: Software expedites singular-spectrum analysis of noisy time series. Eos, Trans. Amer. Geophys. Union, 76, 12, 14, 21.

Dewar, W. K., 2003: Nonlinear midlatitude ocean adjustment. $J$. Phys. Oceanogr., 33, 1057-1081.

Dijkstra, H. A., and M. Ghil, 2005: Low-frequency variability of the large-scale ocean circulation: A dynamical systems approach. Rev. Geophys., 43, RG3002, doi:10.1029/ 2002RG000122.

Feliks, Y., M. Ghil, and E. Simonnet, 2004: Low-frequency variability in the midlatitude atmosphere induced by an oceanic thermal front. J. Atmos. Sci., 61, 961-981.

Folland, C. K., D. E. Parker, and F. E. Kates, 1984: Worldwide marine temperature fluctuations 1856-1981. Nature, 310, 670-673.

Ghil, M., and K. C. Mo, 1991: Intraseasonal oscillations in the global atmosphere. Part I: Northern Hemisphere and Tropics. J. Atmos. Sci., 48, 752-779.

— , and R. Vautard, 1991: Interdecadal oscillations and the warming trend in global temperature time series. Nature, 350, 324-327.

- and Coauthors, 2002a: Advanced spectral methods for climatic time series. Rev. Geophys., 40, 1003, doi:10.1029/ 2000RG000092.

- Y. Yeliks, and L. Sushama, 2002b: Baroclinic and barotropic aspects of the wind-driven ocean circulation. Physica D, 167, $1-35$.

Goodman, J., and J. Marshall, 1999: A model of decadal middlelatitude atmosphere-ocean interaction. J. Climate, 12, 621641.

Hogg, A. M., P. D. Killworth, J. R. Blundell, and W. K. Dewar, 2005: Mechanisms of decadal variability of the wind-driven ocean circulation. J. Phys. Oceanogr., 35, 512-531.

- W. K. Dewar, P. D. Killworth, and J. R. Blundell, 2006: Decadal variability of the midlatitude climate system driven by the ocean circulation. J. Climate, 19, 1149-1166.

Holland, W. R., 1978: The role of mesoscale eddies in the general circulation of the ocean: Numerical experiments using a wind-driven quasigeostrophic model. J. Phys. Oceanogr., 8, 363-392.

Jiang, S., F.-F. Jin, and M. Ghil, 1995: Multiple equilibria, periodic and aperiodic solutions in a wind-driven, double-gyre, shallow-water model. J. Phys. Oceanogr., 25, 764-786.

Keppenne, C. L., and M. Ghil, 1993: Adaptive filtering and prediction of noisy multivariate signals: An application to subannual variability in atmospheric angular momentum. Int. J. Bifurc. Chaos, 3, 625-634.

Kravtsov, S., and A. W. Robertson, 2002: Midlatitude oceanatmosphere interaction in an idealized coupled model. Climate Dyn., 19, 693-711.

$\_, \ldots$, and M. Ghil, 2005a: Bimodal behavior in a baroclinic $\beta$-channel model. J. Atmos. Sci., 62, 1746-1769.

—, D. Kondrashov, and M. Ghil, 2005b: Multiple regression modeling of nonlinear processes: Derivation and applications to climatic variability. J. Climate, 18, 4404-4424.

— W. K. Dewar, P. Berloff, J. C. McWilliams, and M. Ghil, 2006: A highly nonlinear coupled mode of decadal variability in a mid-latitude ocean-atmosphere model. Dyn. Atmos. Oceans, in press.

Kushnir, Y., 1994: Interdecadal variations in North Atlantic sea surface temperature and associated atmospheric conditions. J. Climate, 7, 141-157.

—, and I. Held, 1996: Equilibrium atmospheric response to North Atlantic SST anomalies. J. Climate, 9, 1208-1220.

Mann, M. E., and J. M. Lees, 1996: Robust estimation of background noise and signal detection in climatic time series. Climate Change, 33, 409-445.

Mantua, N. J., S. R. Hare, Y. Zhang, J. M. Wallace, and R. C. Francis, 1997: A Pacific interdecadal climate oscillation with impacts on salmon production. Bull. Amer. Meteor. Soc., 78, 1069-1079.

Mehta, V. M., M. J. Suarez, J. V. Manganello, and T. L. Delworth, 2000: Oceanic influence on the North Atlantic Oscillation and associated Northern Hemisphere climate variations: 1959-1993. Geophys. Res. Lett., 27, 121-124.

Moron, V., R. Vautard, and M. Ghil, 1998: Trends, interdecadal and interannual oscillations in global sea-surface temperatures. Climate Dyn., 14, 545-569.

Plaut, G., and R. Vautard, 1994: Spells of low-frequency variability and weather regimes in the Northern Hemisphere. J. Atmos. Sci., 51, 210-236.

- M. Ghil, and R. Vautard, 1995: Interannual and interdecadal 
variability in 335 years of Central England temperatures. Science, 268, 710-713.

Preisendorfer, R. W., 1988: Principal Component Analysis in Meteorology and Oceanography. Elsevier, $425 \mathrm{pp}$.

Rodwell, M. J., D. P. Rodwell, and C. K. Folland, 1999: Oceanic forcing of the wintertime North Atlantic Oscillation and European climate. Nature, 398, 320-323.

Saravanan, R., 1998: Atmospheric low-frequency variability and its relationship to midlatitude SST variability: Studies using the NCAR Climate System Model. J. Climate, 11, 1386-1404.

Sheremet, V. A., G. R. Ierley, and V. M. Kamenkovich, 1997: Eigenanalysis of the two-dimensional wind-driven ocean circulation problem. J. Mar. Res., 55, 57-92.

Simonnet, E., 2005: Quantization of the low-frequency variability of the double-gyre circulation. J. Phys. Oceanogr., 35, 22682290.

-, M. Ghil, K. Ide, R. Temam, and S. Wang, 2003a: Lowfrequency variability in shallow-water models of the winddriven ocean circulation. Part I: Steady-state solutions. $J$. Phys. Oceanogr., 33, 712-728.

$-, \ldots,-,-$, and $\_$, 2003b: Low-frequency variability in shallow-water models of the wind-driven ocean circulation.
Part II: Time-dependent solutions. J. Phys. Oceanogr., 33, 729-752.

,-- , and H. A. Dijkstra, 2005: Homoclinic bifurcations in the quasi-geostrophic double-gyre circulation. J. Mar. Res., 63, 931-956.

Speich, S., H. Dijkstra, and M. Ghil, 1995: Successive bifurcations in a shallow-water model, applied to the wind-driven ocean circulation. Nonlinear Processes, Geophys., 2, 241-268.

Thomson, D. J., 1982: Spectrum estimation and harmonic analysis. IEEE Proc., 70, 1055-1096.

— 1990: Quadratic-inverse spectrum estimates: Application to paleoclimatology. Philos. Trans. Roy. Soc. London, 332A, 539-597.

Vautard, R., and M. Ghil, 1989: Singular spectrum analysis in nonlinear dynamics, with applications to paleoclimatic time series. Physica D, 35, 395-424.

— , P. Yiou, and M. Ghil, 1992: Singular-spectrum analysis: A toolkit for short, noisy chaotic signals. Physica D, 58, 95-126.

Wunsch, C., 1999: The interpretation of short climate records, with comments on the North Atlantic and Southern Oscillations. Bull. Amer. Meteor. Soc., 80, 245-255. 